\title{
Impacto del uso de dispositivos móviles en el aprendizaje de estudiantes adolescentes
}

\section{Impact From The Use of Mobile Devices In Learning Among Adolescent Students}

\author{
Gloria Yaneth Calderón Loeza \\ https://orcid.org/0000-0002-3320-5606 \\ Universidad Autónoma de Yucatán \\ e.gloriacalderon@correo.uady.mx \\ México
}

\author{
Pedro Sánchez Escobedo \\ https://orcid.org/0000-0002-0564-3502 \\ Universidad Autónoma de Yucatán \\ psanchez@correo.uady.mx
}

\section{Resumen:}

En virtud de la controversia que representan los dispositivos móviles (DMs) en el ámbito escolar de los estudiantes jóvenes, en la presente revisión sistemática reunimos la literatura más reciente al respecto. A través de la búsqueda en las bases de datos Web of Science, Academic Search Complete de Ebsco, Scopus y Eric, se categorizaron y sintetizaron los principales temas de investigación y su contenido. Tras la aplicación de los criterios de inclusión y exclusión, fueron seleccionados 15 estudios de las 121 iniciales; para darle validez y confiabilidad se utilizaron los criterios de Caldwell (2011) y se comprobó la calidad metodológica en 13 de ellos. La mayoría de los estudios demuestran un impacto positivo en el aprendizaje de los estudiantes de secundaria. Se concluye la persistencia de la brecha digital entre los estudiantes de este nivel en el mundo y la tendencia de estudios con enfoque cualitativo en los países ya estudiados. Se sugieren estudios exploratorios en poblaciones poco estudiadas como las de América y Latinoamérica. Se espera un cambio positivo en el impacto de los DMs tras la pandemia por el COVID-19.

Palabras clave: dispositivo móvil; teléfono inteligente; estudiantes; aprendizaje.

\begin{abstract}
:
In view of the controversy of mobile devices (MDs) in the school's environment of young students, this systematic review gathers the most recent literature on the topic. Through searches on the databases Web of Science, EBSCO's Academic Search Complete, Scopus and Eric, we categorized and synthesized the main research topics and their content. After applying the inclusion and exclusion criteria, 15 studies were selected from the initial 121; Caldwell's (2011) criteria were used to ensure validity and reliability, while the methodological quality was verified in 13 of the studies. Most of the research papers included showed a positive impact on learning among high school students. We conclude that the digital divide persists worldwide among students at this level, and that there is a trend for qualitative focus in countries already studied. We suggest exploratory studies in less studied populations such as those of the countries of America and Latin America. We expect a positive change of the impact of MDs after the COVID-19 pandemic.
\end{abstract}

Keywords: mobile device, smartphone, students, learning.

Recibido: 06/10/2020 | Aceptado: 09/11/2020 | Publicado: 08/01/2021

| pág. 31 - 50 
Impacto del uso de dispositivos móviles en el aprendizaje de estudiantes adolescentes

\section{| Introducción}

Hoy en día las tecnologías, el uso de la Internet y los Dispositivos Móviles (DMs) forman parte de la cotidianidad de los jóvenes estudiantes y los han adoptado como parte de su estilo de vida (Fumero, 2016; Soriano, 2018). A nivel mundial existen 5.1 billones de usuarios de teléfonos inteligentes y más de 4 millones de usuarios de Internet, con un porcentaje de uso del $58.7 \%$, que aumentó tras la pandemia COVID-19 (2019-2020) en el mundo. El uso de los smartphones pasó de un 52 a un 79\% (Kemp, 2020). Con el aumento del recurso tecnológico a nivel mundial, se empieza a consolidar una de las propuestas del Fondo de las Naciones Unidas para la Infancia (UNICEF) y la Organización de las Naciones Unidas (ONU) sobre la promoción de la utilización del Internet como un medio para impulsar el desarrollo y competencias de vida de los niños y jóvenes, invitando a los gobiernos para facilitar de forma equitativa y general el acceso a Internet y a las tecnologías digitales (Organización de las Naciones Unidas para la Educación, la Ciencia y la Cultura [UNESCO], 2017; Organización de las Naciones Unidas, 2014).

\section{| Aspectos Teóricos}

La popularidad del smartphone entre los jóvenes y la prevalencia de mejores promedios entre los usuarios frecuentes demuestran su potencial como herramienta de aprendizaje, que debe ser más utilizado por los profesores como un aliado para favorecer procesos colaborativos de enseñanzaaprendizaje (Epalza, et al., 2014). En la misma línea, Basantes (2017) afirma que la utilización de los DMs constituyen un potencial para el desarrollo del aprendizaje de los estudiantes, impulsando su motivación, la satisfacción de interacción entre compañeros y docentes, además de estimular el pensamiento crítico y reflexivo. En un meta análisis realizado por 
Xie et al. (2018), se reportó que el uso de pantallas táctiles podría facilitar el aprendizaje a los niños menores de cinco años de edad, sobre todo cuando se desarrollan actividades relacionadas al STEM (Ciencia, Tecnología, Ingenieria y Matemáticas). Por su parte, Herodotou (2018) plantea, en una revisión sistemática, que los DMs y aplicaciones móviles demuestran efectos benéficos en el aprendizaje de menores de cinco años de edad en 14 de los estudios revisados; los cuales, son utilizados como una herramienta para el desarrollo del vocabulario y la lectoescritura, el desarrollo de habilidades STEM y sobre la resolución de problemas, la colaboración y el desarrollo de habilidades motoras finas. En cuatro estudios se reportaron resutados mixtos (benéficos y perjudiciales), y solo uno mostró efectos perjudiciales correspondientes a la comprensión de texto de libros electrónicos versus tradicionales, con el acompañamiento de los padres.

Por otro lado se evidencian efectos controversiales vinculados al uso de los DMs, como es el caso de Cuba Alvarado (2016), sobre el uso de los celulares con conexión a la Internet y rendimiento académico en estudiantes universitarios en el que se expuso que éstos perciben que el uso del teléfono inteligente, si bien no afecta en su promedio académico, sí afecta en el tiempo que dedican para estudiar o hacer actividades académicas; es decir, lo perciben como beneficioso por un lado y perjudicial por otro. DíazVicario et al. (2019) confirman que el uso de las Tecnologías de la Información y Comunicación (TIC) en el ámbito académico pueden generar distracciones en clase $\mathrm{y}$ propiciar la disminución del tiempo dedicado al estudio. Por su parte, en una revisión sistemática, Carbonell et al. (2012) reportaron que el uso excesivo de las TIC puede generar un impacto negativo en el ámbito académico de los jóvenes, entre ellos la vinculación al fracaso escolar. La prevalencia registrada por el consumo problemático de la Internet en este estudio varía del $3.7 \%$ hasta el $9.9 \%$ y existe relación con la cantidad de tiempo de conexión. Tomando en cuenta que la edad a la que se le asigna a un adolescente la autonomía para portar un teléfono inteligente es alrededor de los 10 a 12 años (De-Sola, et al., 2017; Román, 2017), se infiere que cerca del $10 \%$ de los adolescentes a partir de los 
10 años de edad podrían presentar alguna afectación en el aprendizaje secundario al uso abusivo de los DMs. Aunque se cubre el aspecto social como efecto perjudicial, no hay suficiente información en el impacto en el aprendizaje de los jóvenes estudiantes representada en revisiones sistemáticas. Esta percepción de abuso al Dispositivo Móvil (DM) también es señalada por los profesores de secundaria (Nikolopoulou, 2020).

\section{| Aportaciones}

La presente revisión sistemática (RS) pretende identificar las investigaciones existentes más recientes sobre el tema y analizarlas críticamente, identificando los efectos benéficos o perjudiciales que producen los DMs en el aprendizaje de los jóvenes estudiantes. Como se mostró previamente, existe literatura que demuestra la preocupación generalizada por este grupo poblacional pero no existe alguna RS que se enfoque específicamente a los efectos en el aprendizaje en la población de estudiantes adolescentes. Por lo que este es el vacío de conocimiento al que aportaremos respondiendo la siguiente pregunta de investigación:

\section{Pregunta de investigación}

¿Cuál es el impacto del uso del dispositivo móvil en el aprendizaje de los estudiantes adolescentes?

\section{Objetivo}

Sintetizar y clasificar la evidencia del impacto (positivo, negativo, neutral) en el aprendizaje de los estudiantes adolescentes por el uso del dispositivo móvil.

\section{Método}

Una revisión sistemática es un resumen de la literatura de investigación que se centra en una sola pregunta, el cual trata de identificar, seleccionar, evaluar y sintetizar toda la evidencia de investigación de alta calidad relevante para esa pregunta (Bettany-Saltikov, 2013). En principio se planteó la pregunta de investigación y el marco conceptual del diseño; luego, se utilizó el formato PEO (Patient, Exposure and Outcome) para identificar las partes de la pregunta, lo cual se describe de la siguiente forma:

Estudiantes de secundaria (P) - Uso del dispositivo móvil (E) - Impacto en el aprendizaje (0) 
En segundo término, refiriendo al marco conceptual, emergieron los criterios de inclusión y exclusión, los cuales se presentan a continuación.
- Estudiantes Universitarios o de mayor grado

- Adolescentes con algún trastorno del desarrollo

\section{Criterios de inclusión}

- Estudios empíricos en estudiantes de secundaria que en el título, resumen y palabras clave contengan al menos las palabras en inglés: student, learning, mobile device

- Información reciente de los últimos cinco años (estudios comprendidos del 2016 hasta marzo del 2020)

- Estudios con participantes entre 12 años y 18 años que cursen la escolaridad secundaria

- Publicaciones en revistas arbitradas

- Lenguajes inglés y español

- Artículo completo con libre acceso

\section{Criterios de exclusión}

- Libros, capítulos de libro, notas, conferencias

- Estudiantes de primaria o de menor grado
Una vez formulada la pregunta e integrados los criterios de inclusión y exclusión, se realizó una búsqueda de alcance, con el fin de encontrar alguna revisión sistemática similar a la planeada. Al descartarse, se procedió a realizar la búsqueda con palabras clave relacionadas al uso de los DMs, como se identificó previamente en la literatura, y se colocaron sinónimos del término abuso, como son: uso excesivo, uso problemático, uso adictivo, uso patológico, uso dependiente. Se incluyeron los términos laptop y smartphone a los DMs, ya que son los dispositivos que utilizan los estudiantes con más frecuencia para fines escolares (Díaz-Vicario et al., 2019). Respecto a los factores del aprendizaje asociados al uso de los DMs, fue preciso colocar sinónimos o palabras relacionadas al aprendizaje como son: rendimiento académico, escuela, educación, formación intelectual, conocimientos, habilidades escolares, actitudes escolares, 
estrés académico, desarrollo de competencias. Se realizó la cadena de búsqueda en inglés y español en las bases de datos seleccionadas.

\section{Bases de datos}

Se seleccionaron cuatro bases de datos de impacto internacional, relacionadas al área de la educación como son Web of Science (WOS), EBSCO Host, Scopus y Education Resources Information Center (ERIC). Se registraron los criterios de búsqueda ya señalados, definiendo en los buscadores de cada plataforma que los términos se encontraran en los apartados de palabras clave, título y resumen. En la primera fase de búsqueda se examinaron los títulos y resúmenes del total. Los estudios recuperados ( $\mathrm{n}=106)$ fueron seleccionados sistemáticamente $\mathrm{y}$ con la aplicación de los criterios de inclusión o exclusión, quedaron como resultado 17 artículos en total. La segunda fase consistió en la lectura del texto completo de cada artículo identificado.

\section{Aplicación de las directrices de Caldwell}

Para darle validez y confiabilidad a los estudios se siguieron los marcos y directrices de Caldwell et al. (2011), obtenido de Bettany-Saltikov (2013), tomando en cuenta los siguientes criterios:

1. El título se refleja en el contenido

2. Los autores muestran credibilidad

3. El resumen presenta los componentes clave

4. Se identifica claramente el propósito del estudio

5. La revisión de la literatura es exhaustiva y actualizada

6. El objetivo es claro

7. Se evidencian las consideraciones éticas

8. Se identifica la metodología y es justificada

9. Los resultados son claros y coherentes

10. La conclusión es comprensiva

Además, para minimizar el riesgo de sesgo, se utilizaron las siguientes pautas de la declaración PRISMA (Preferred Reporting ítems for Systematic reviews and MetaAnályses) (Urrútia y Bonfill, 2010):

1. La arbitrariedad de las publicaciones. Este criterio 
fue colocado en el proceso de selección de los estudios como parte de los criterios de inclusión.

2. La validez interna de los datos. Corroborada en la sección de métodos de cada artículo revisado; en caso de la omisión de la presente o presentar información incompleta, se excluiría el estudio.

Todos los autores fueron claramente identificados en el artículo, todos mencionaban la Universidad o Institución de donde provenían y un correo electrónico de contacto. Solo un estudio resumió parte de su experiencia en el tema, en un apartado independiente (Villani et al., 2018). Respecto a si el resumen presenta los componentes clave, la identificación del propósito del estudio, la revisión de la literatura exhaustiva, actualizada y objetivo claro, todos los estudios los cumplen. Las consideraciones éticas fueron la debilidad de los estudios, ya que $60 \%$ de ellos no las mencionan; este aspecto se encontró bien definido en un solo estudio (Chen, 2020). Otros señalaron en la metodología el carácter voluntario de la participación de los estudiantes (Fernández-Soriano, et al., 2019; Villani et al., 2018), el cuidado de la privacidad (Kennedy, 2018; Vázquez et al., 2016) y el retiro de su participación en el momento que lo deseen (Ally, et al., 2017). En los estudios cuantitativos se identifica la metodología y está justificada de acuerdo con diseño de estudio. Caso contrario se presenta en los estudios cualitativos donde no se identifica claramente la técnica del procesamiento y validez de información (triangulación u otra técnica estadística cualitativa). Tampoco se describe el papel del investigador. Se definen resultados y conclusiones comprensibles en todos los estudios. Al final fueron 15 estudios seleccionados. Dos artículos fueron eliminados. El primero fue de un programa gubernamental, donde el DM formaba parte de la infraestructura a evaluar (no cumplía el constructo en el aprendizaje), y el segundo debido a que las características de los participantes eran de un grupo con dificultades en el aprendizaje (criterios de exclusión), los cuales no fueron señalados previamente en el 
título ni el resumen. La descripción se cuatro fases: identificación, cribado, presenta en un diagrama de flujo con elegibilidad y síntesis (ver figura 1).

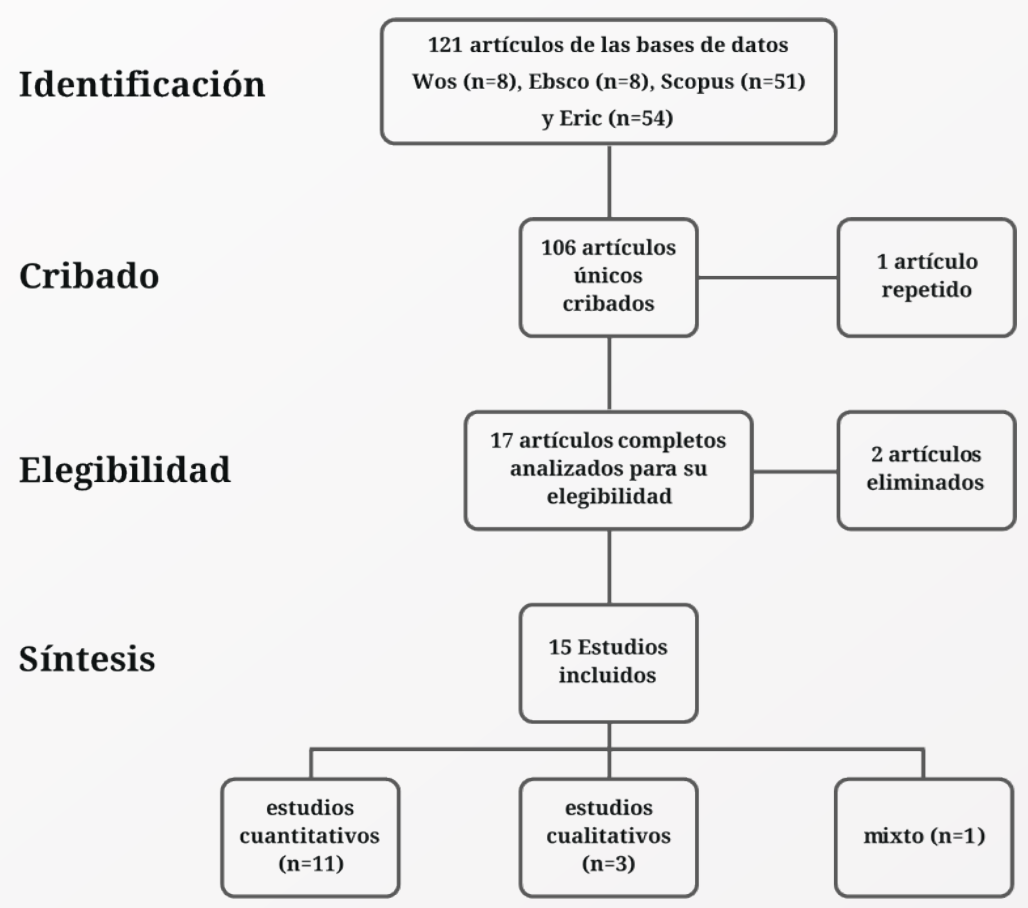

Figura 1: Diagrama de flujo de la revisión sistemática.

\section{Resultados}

De los 15 estudios revisados, siete se de Ebsco Host versión completa, tres obtuvieron de la plataforma Eric, dos de Scopus y tres de WoS (ver figura 2).

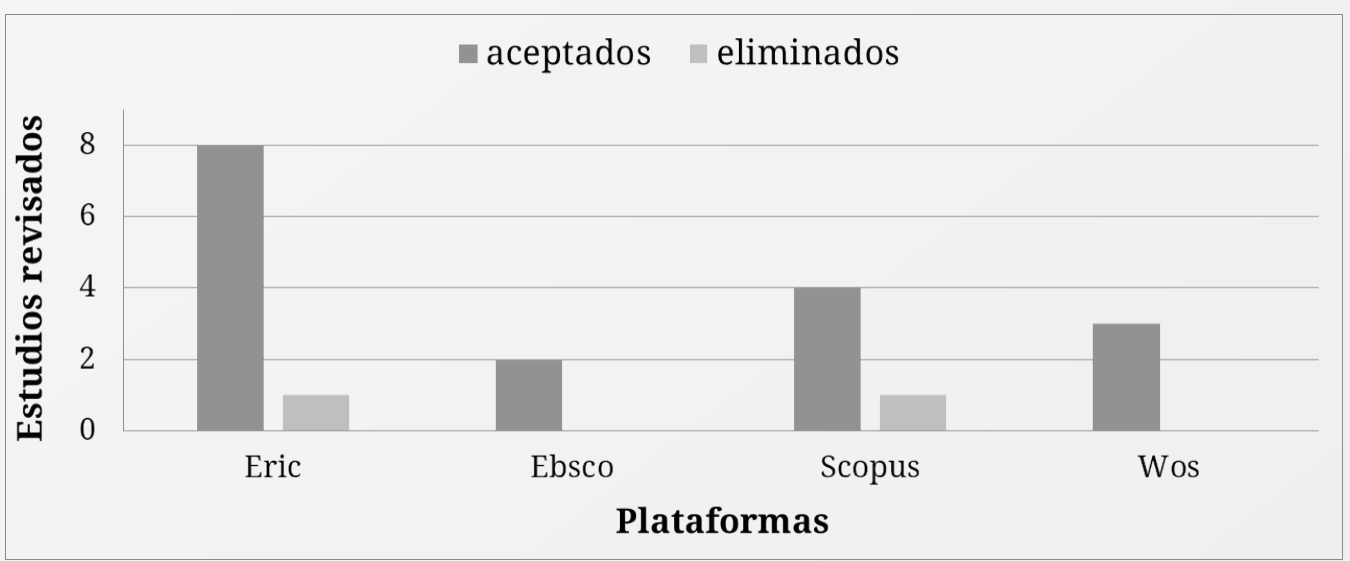

Figura 2: Lista de artículos seleccionados y eliminados según la base de datos consultada. 
El continente que más estudios tres, seguido de Pakistán con dos. aportó fue Asia con nueve Se seleccionó el periodo del 2016 (Tailandia, Pakistán, Japón, Turquía, a marzo 2020, comprendiendo la Bangladesh, Taiwán, Indonesia, literatura de los cinco años más Hong Kong); en segundo lugar, se encontraron cinco del continente europeo (Dinamarca, España, Italia) y en tercer lugar, con uno, Oceanía (Reino de Tonga). El país que más estudios aportó fue España con recientes, previo al impacto escolar de la pandemia COVID-19 en México y en el mundo. El número de estudios en el periodo se distribuyó de la siguiente forma (ver figura 3).

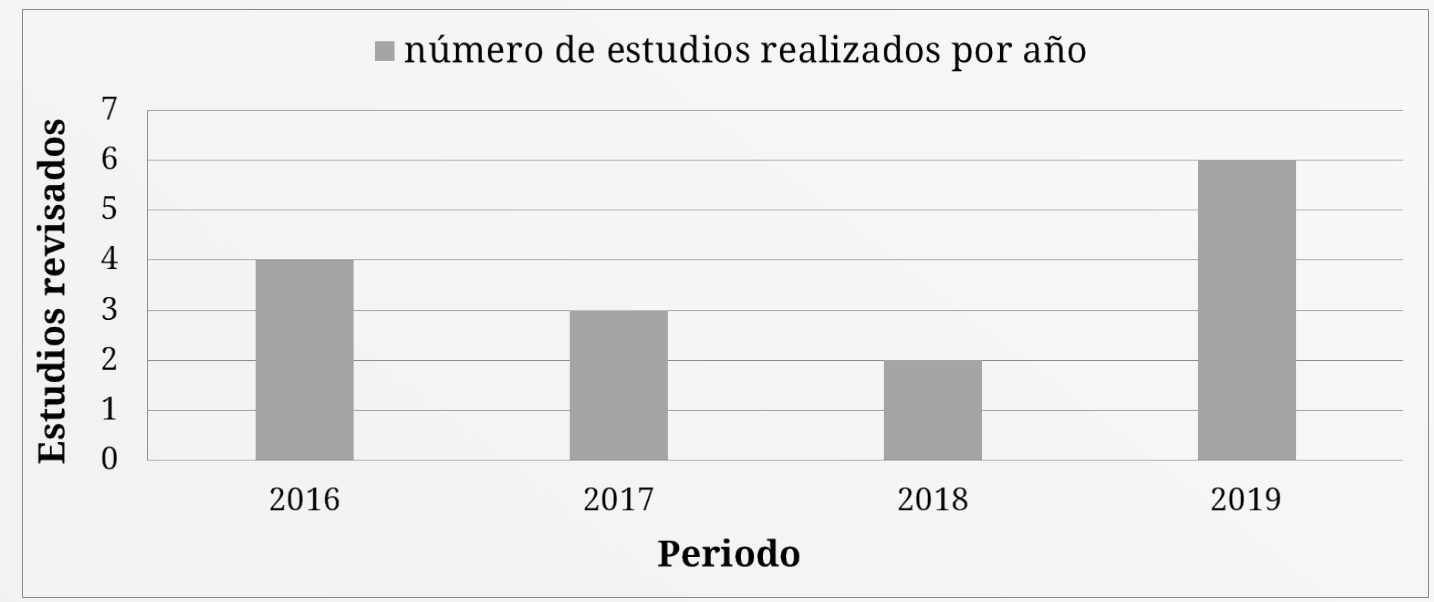

Figura 3: Número de estudios realizados por año.

\section{Dispositivo móvil}

El DM más utilizado en la los estudios utilizó además de presente RS fue la tablet, seguido del smartphone y la laptop. En cinco estudios se incluyeron más de dos dispositivos electrónicos; la combinación smartphonetablet fue la más común. Uno de DM la grabadora de video digital y Lego Mindstorms NTX por la utilidad en el laboratorio de física e hizo comparaciones entre los dispositivos electrónicos como herramienta de aprendizaje (Liu, et al., 2017) (ver figura 4). 


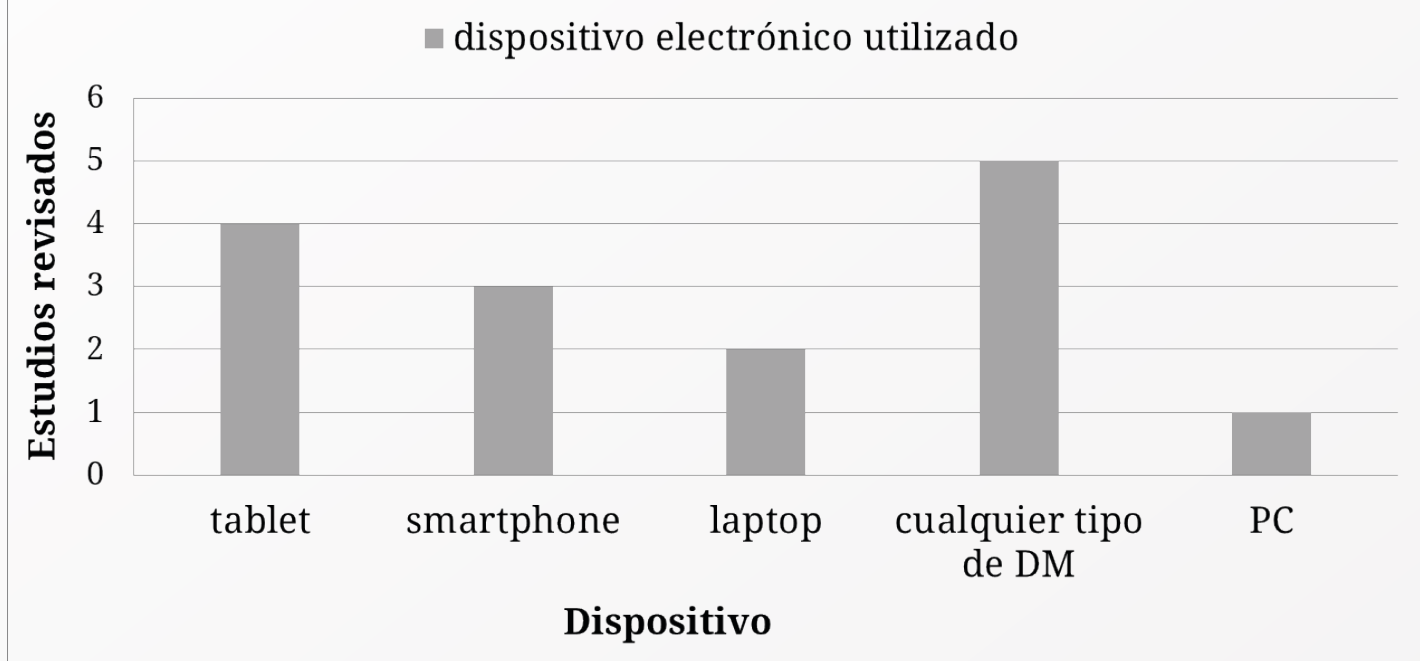

Figura 4: Tipo de DM utilizado.

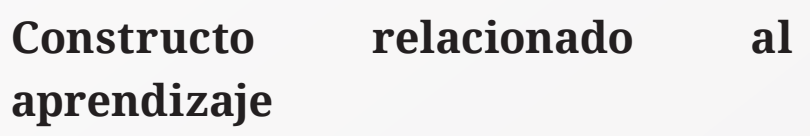

El constructo relacionado al a nivel general se refleja de la aprendizaje evaluado o relacionado siguiente manera (ver figura 5). con el uso del DM en este estudio

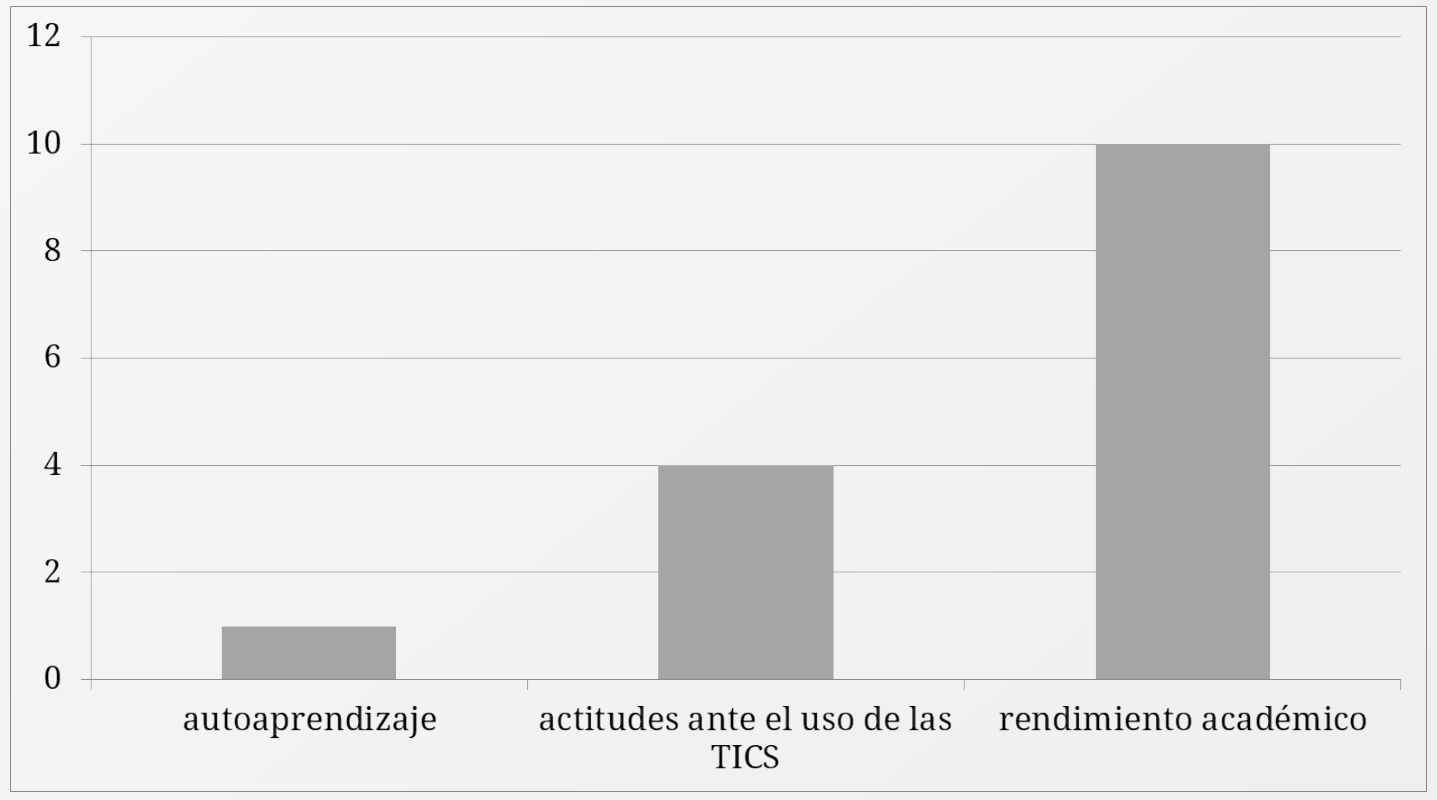

Figura 5: Constructo de aprendizaje. 


\section{Participantes}

La edad mínima registrada fue de nueve años (Fernández-Soriano et al., 2019), y la máxima, de 21 años (Hans, et al.,2016), con un promedio de 15 años en los 15 estudios revisados. En uno de los estudios, profesores y padres de familia fueron incluidos como participantes en entrevistas semi-estructuradas debido al requerimiento de triangulación (Vázquez et al., 2016). La escolaridad en todos los estudios revisado fue de secundaria, a excepción de uno que incluyó, además de la escolaridad secundaria, los dos últimos grados de primaria y los dos primeros grados de preparatoria (Fernández-Soriano et al., 2019). El lugar donde se realizaron fue predominantemente dentro de escuelas públicas (80\%), dos en escuelas privadas con nivel socioeconómicos medio-alto (Fernández-Soriano et al., 2019; Liu et al., 2017) y una escuela de música popular (Chen, 2020). La cantidad de muestra total en los 13 estudios fue de 178,964 participantes; la muestra más alta fue de 175,493 participantes registrado por Mora, et al. (2018), y la más baja con 12 (Vázquez Calvo y Cassany, 2016); dos estudios no especificaron la cantidad de participantes (Hasan, et al., 2016; Meyer, 2016).

\section{Diseño metodológico}

Once de los estudios fueron de tipo cuantitativo, tres cualitativos (Kennedy, 2018; Meyer, 2016; Vázquez et al., 2016) y uno se identificó como mixto (Ally et al., 2017). Se utilizaron diversas técnicas de recogida de datos; el más frecuente en los estudios cuantitativos fue la aplicación de encuestas, de los cuales nueve fueron aplicados directamente con los alumnos, dos se realizaron en línea (Chen, 2020; Villani et al., 2018). En ocho estudios se diseñó su propio cuestionario, que fue tipo Likert; todos colocaron registros de validez interna y externa del instrumento, excepto uno (Hans et al., 2016). En uno de los estudios se utilizó grupo control al ser experimental comparativo (Pramuda, et al., 2019), y en otro se hicieron comparaciones entre grupos con tres 
diferentes DMs (Liu et al., 2017). Solo un estudio de enfoque cualitativo se identificó como diseño etnográfico de múltiples ubicaciones (Meyer, 2016), uno más fue estudio de caso (Vázquez et al., 2016), y en otro no se identificó el diseño, pero se infiere una investigación participativa (Kennedy, 2018). Respecto a los estudios cualitativos, se utilizaron técnicas de entrevista, grupos focales, entrevista semi-estructurada y observación. En el caso de la etnografía se utilizaron además de la observación, otros recursos como son las grabaciones de voz y video, notas, fotos de enseñanza y escenarios de aprendizaje.

\section{Procesamiento estadístico}

En los estudios cuantitativos se utilizaron técnicas de procesamiento de datos como es el caso de estadística descriptiva e inferencial, además de correlaciones y bivarianza. En los estudios cualitativos se identificó triangulación de datos (Meyer, 2016; Vázquez et al., 2016), con entrevistas a alumnos, profesores y padres. Uno de los estudios se basó solamente en narrativas de los alumnos y no se especifica técnica de procesamiento de datos (triangulación, redes semánticas, lista de verificación, estadística cualitativa). Ninguno de los estudios cualitativos presenta el perfil del investigador dentro de su estudio.

\section{Impacto del Dispositivo Móvil}

Para definir si el impacto del DM era positivo, negativo o neutro, se revisaron los resultados de los estudios que debían contener validez en su diseño metodológico y estadístico, en concordancia con el propósito y los resultados, lo cual se pudo confirmar en todos los estudios, excepto en el estudio de Hans et al., (2016) puesto que su instrumento no demuestra validez interna o externa descrito en el artículo; tampoco se consideró el estudio de Howlett y Waemusa, (2019) debido a que no demostró evidencia de validación de los resultados con técnicas apropiada. A continuación, se expresan los resultados de los estudios (ver tabla 1). 
Tabla 1.

Tipo de impacto en el aprendizaje por estudio

\begin{tabular}{|c|c|c|}
\hline Autores & Conclusión & $\begin{array}{l}\text { Tipo de } \\
\text { impacto }\end{array}$ \\
\hline $\begin{array}{c}\text { (Howlett y } \\
\text { Waemusa, 2019) }\end{array}$ & $\begin{array}{l}\text { Los DMs favorecen el aprendizaje del idioma inglés, la satisfacción } \\
\text { del alumno en el entorno escolar y el aprendizaje autónomo }\end{array}$ & Positivo \\
\hline (Jan, 2018) & $\begin{array}{l}\text { La alfabetización digital, el uso de tabletas y teléfonos inteligentes, } \\
\text { la capacitación en el uso de la computadora y el número de años que } \\
\text { usan las TIC tienen una correlación significativa con la actitud hacia } \\
\text { los DMs }\end{array}$ & Positivo \\
\hline (Meyer, 2016) & $\begin{array}{l}\text { El uso de la tecnología móvil para acceder e interactuar con los } \\
\text { materiales de aprendizaje electrónico benefician el rendimiento } \\
\text { académico de los estudiantes y mejoran sus habilidades de TIC para } \\
\text { prepararlos para la era tecnológica }\end{array}$ & Positivo \\
\hline $\begin{array}{l}\text { (Sirakaya y Kiliç } \\
\text { Çakmak, 2017) }\end{array}$ & $\begin{array}{l}\text { Los estudiantes muestran actitudes positivas hacia las aplicaciones } \\
\text { con realidad aumentada }\end{array}$ & Positivo \\
\hline $\begin{array}{l}\text { (Ally et al., } \\
\text { 2017) }\end{array}$ & $\begin{array}{l}\text { El uso de las tablets aumenta el rendimiento académico de los } \\
\text { estudiantes }\end{array}$ & Positivo \\
\hline $\begin{array}{l}\text { (Mora et al., } \\
\text { 2018) }\end{array}$ & $\begin{array}{l}\text { El programa One Lap Top por Child que promueve el uso de la } \\
\text { lap tiene un efecto negativo en el rendimiento académico de los } \\
\text { estudiantes }\end{array}$ & Negativo \\
\hline $\begin{array}{l}\text { (Hasan et al., } \\
\text { 2016) }\end{array}$ & $\begin{array}{l}\text { Las aplicaciones móviles a través de los smartphones proporcionan } \\
\text { una percepción positiva para mejorar el sistema de aprendizaje en } \\
\text { los estudiantes }\end{array}$ & Positivo \\
\hline (Liu et al., 2017) & $\begin{array}{l}\text { El smartphone como herramienta de aprendizaje en el laboratorio } \\
\text { de física supera en preferencia al video y NTX. Solo demostró } \\
\text { significancia estadística en el aprendizaje de modelado }\end{array}$ & Positivo \\
\hline $\begin{array}{l}\text { (Villani et al., } \\
\text { 2018) }\end{array}$ & $\begin{array}{l}\text { Se identificaron tres grupos con alta, moderada y baja aceptación de } \\
\text { tablet PC. Estudiantes que tienen un mayor nivel de aceptación son } \\
\text { aquellos que usan más la herramienta tanto para el aprendizaje como } \\
\text { para la comunicación }\end{array}$ & Neutro \\
\hline $\begin{array}{l}\text { (Pramuda et al., } \\
\text { 2019) }\end{array}$ & $\begin{array}{l}\text { RPO puede aumentar la autoconfianza y el deseo de aprender, pero no } \\
\text { genera diferencia estadística entre comparado al método tradicional }\end{array}$ & Neutro \\
\hline (Chen, 2020) & $\begin{array}{l}\text { Los estudiantes de instrumentos y los estudiantes de instrumentos } \\
\text { mostraron un aumento significativo en la motivación cuando usan } \\
\text { las tabletas móviles }\end{array}$ & Positivo \\
\hline $\begin{array}{l}\text { (Fernández- } \\
\text { Soriano et al., } \\
\text { 2019) }\end{array}$ & $\begin{array}{l}\text { Los estudiantes que acceden al LMS mejoran significativamente el } \\
\text { rendimiento académico. El tipo de dispositivo y el sistema operativo } \\
\text { influyen en la cantidad de asignaturas aprobadas }\end{array}$ & Positivo \\
\hline
\end{tabular}




\section{| Conclusiones}

Los continentes que aportan más estudios a nivel mundial son Asia y Europa. Esto es similar a la literatura revisada previamente (Díaz-Vicario et al., 2019), lo que nos lleva a las siguientes interrogantes ¿Qué está sucediendo en América y Latinoamérica? ¿Cómo influye la tecnología en el aprendizaje de los estudiantes latinoamericanos?

La combinación de uso del smartphone-tablet fue la más común para los estudiantes de secundaria en esta RS, también fue previamente identificado por otros autores (Díaz-Vicario et al., 2019; Basantes et al., 2017), quienes reportan la preferencia del estudiante por esta combinación de herramientas académicas.

Si bien, la utilización de los DMs en el aprendizaje es promovida por instituciones de gran relevancia a nivel mundial como la ONU y la Unesco, para “asegurar una educación de calidad inclusiva y equitativa y promover oportunidades de aprendizaje permanente para todos" (UNESCO, 2017), este recurso aún no está distribuido de forma global. En el presente estudio se evidencia que las escuelas participantes fueron cubiertas de la siguiente manera: escuelas con recursos socioeconómicos medios a altos, escuelas privadas que garantizan la disposición de los DMs o bien, escuelas con programas donde se otorgó un DM por instituciones gubernamentales. Las clases de inglés u otro idioma, el laboratorio de física y música fueron las áreas más comunes de aplicación del DM como herramienta de aprendizaje.

Una de las deficiencias generales que mostraron los estudios revisados fueron las consideraciones éticas, las cuales fueron nombradas someramente en algunos casos y en otros omitidas. Otro aspecto para notarse en la metodología de los estudios cualitativos es el proceso de validación de los resultados en el cual no se define con precisión la técnica, y en el caso de la etnografía, el tiempo que llevó la recolección de datos (Stake, 2010); aun así, el estudio de Kennedy (2018) como narrativa, muestra hallazgos de un impacto negativo en el desempeño académico de los estudiantes, lo que podría considerarse una perspectiva 
novedosa al tema de investigación, por lo que es preciso promover este tipo de enfoque.

Más del $70 \%$ de los estudios revisados presentan un impacto positivo en el aprendizaje; 20\%, un impacto neutro; y menos del $10 \%$, un impacto negativo. Esto es similar a lo reportado en estudios donde relacionan el impacto de los DMs en niños (Herodotou, 2018; Xie et al. 2018). El impacto positivo de los DMs en el aprendizaje sigue superando a los negativos o neutros. De manera general, el impacto fue clasificado en tres grupos: el rendimiento académico, actitud hacia el dispositivo y la autonomía en el aprendizaje. Se prevé un gran cambio en este impacto tras la pandemia COVID-19 en el mundo.
Esta RS presenta algunas limitaciones respecto al tipo de estudios revisados; si se expandiera a conferencias o notas podría obtener información más actualizada y numerosa. Además, se limitó a la literatura de acceso abierto por lo que hay artículos que no pudieron analizarse. Este estudio da apertura a futuras líneas de investigación en este tema, sobre todo a países de Latinoamérica para iniciar investigaciones exploratorias, y a los países desarrollados, a ampliar su experiencia utilizando alternativas de diseños metodológicos para profundizar en el impacto que los DMs están generando en los estudiantes. 


\section{|Referencias}

Ally, M., Balaji, V., Abdelbaki, A. \& Cheng, R. (2017). Use of Tablet Computers to Improve Access to Education in a Remote Location. Journal of Learning for Development, 4(2), 221-228. https://files.eric.ed.gov/fulltext/ EJ1149172.pdf

Basantes, A. V., Naranjo, M. E., Gallegos, M. C. \& Benítez, N. M. (2017). Los dispositivos móviles en el proceso de aprendizaje de la facultad de educación ciencia y tecnología de la universidad técnica del norte de ecuador. Formacion Universitaria, 10(2), 79-88. https://doi.org/10.4067/ S0718-50062017000200009

Bettany-Saltikov, J. (2013). How to do a Systematic Literature Review in Nursing. A Step-by-Step Guide. Nurse Education in Practice, 13(3), e9. https://doi. org/10.1016/j.nepr.2012.12.004

Carbonell, X., Fúster, H., Chamarro, A. \& Oberst, U. (2012). Adicción a internet y móvil: Una revisión de estudios empíricos Españoles. Papeles del Psicólogo, 33(2), 82-89. http://www.redalyc.org/articulo. oa?id=778/77823407001

Chen, J. C. W. (2020). Mobile composing: Professional practices and impact on students' motivation in popular music. International Journal of Music Education, 38(1), 147-158. https://doi.org/10.1177/0255761419855820

Cuba Alvarado, C. P. (2016). Uso de los celulares con internet y rendimiento académico de estudiantes universitarios. [Tesis de Licenciatura inédita]. Universidad de Lima, Perú. http://doi.org/10.26439/ulima.tesis/4761

De-Sola, J., Talledo, H., Rodríguez de Fonseca, F. \& Rubio, G. (2017). Prevalence of problematic cell phone use in an adult population in Spain as assessed by the Mobile Phone Problem Use Scale (MPPUS). PLoS ONE, 12(8), 1-17. https://doi.org/10.1371/journal.pone.0181184

Díaz-Vicario, A., Mercader, J. \& Gairín Sallán, J. (2019). Uso problemático de las TIC en adolescentes. Revista Electrónica de Investigación Educativa, 21(1), 1. https://doi.org/10.24320/redie.2019.21.e07.1882 
Epalza, M. P., Paternina, J., Río, D. \& Santiago, D. M. (2014). Uso Smartphones. Salud Uninorte, 30(3), 335-346. https://www.redalyc.org/articulo. oa? id $=817 / 81737153008$

Fernández-Soriano, F. L., López, B., Martínez-España, R., Muñoz, A. \& Cantabella, M. (2019). Use of computing devices as sensors to measure their impact on primary and secondary students' performance. Sensors (Switzerland), 19(14), 3226. https://doi.org/10.3390/s19143226

Fumero A. (2016). Jóvenes Z. Fundación Cibervoluntarios, 1-15. https://www. researchgate.net/publication/310057399_Jovenes_y_Generacion_Z/citations

Hans, T., Sopu, Chisaki, Y. \& Usagawa, T. (2016). Use of facebook by secondary school students at Nuku'alofa as an indicator of E-readiness for E-learning in the Kingdom of Tonga. International Review of Research in Open and Distance Learning, 17(4), 203-223. https://doi.org/10.19173/ irrodl.v17i4.2333

Hasan, N., Ashraf, M. M., Abdullah, A. \& Murad, M. W. (2016). Introducing mobile internet as a learning assistant for secondary and higher secondary students. The Journal of Developing Areas, 50(5), 41-55. https://doi.org/10.1353/jda.2016.0061

Herodotou, C. (2018). Young children and tablets: A systematic review of effects on learning and development. Journal of Computer Assisted Learning, 34(1), 1-9. https://doi.org/10.1111/jcal.12220

Howlett, G. y Waemusa, Z. (2019). 21 st century learning skills and autonomy: Students' perceptions of mobile devices in the Thai EFL context. Teaching English with Technology, 19(1), 72-85. https://files.eric.ed.gov/ fulltext/EJ1204626.pdf

Jan, S. (2018). Investigating the relationship between students' digital literacy and their attitude towards using ICT. International Journal of Educational Technology, 5(2), 26-34. https://ecommons.aku.edu/ pakistan_ied_pdck/304 
Kemp, S. (2020). Digital in 2020. https://wearesocial.com/digital-2020

Kennedy, O. (2018). Examining student perceptions about smartphones to understand lack of acceptance of mobile-assisted language learning. Future-proof CALL: language learning as exploration and encounters short papers from EUROCALL 2018, 137-141. https://doi.org/10.14705/ rpnet.2018.26.826

Liu, C. Y., Wu, C. J., Wong, W. K., Lien, Y. W. \& Chao, T. K. (2017). Scientific modeling with mobile devices in high school physics labs. Computers and Education, 105, 44-56. https://doi.org/10.1016/j.compedu.2016.11.004

Meyer, B. (2016). Mobile devices and spatial enactments of learning: IPads in lower secondary schools. Proceedings of the 12th International Conference on Mobile Learning, 3-10. https://files.eric.ed.gov/fulltext/ ED571444.pdf

Mora, T., Escardíbul, J. O. \& Di Pietro, G. (2018). Computers and students' achievement: An analysis of the One Laptop per Child program in Catalonia. International Journal of Educational Research, 92, 145-157. https://doi.org/10.1016/j.ijer.2018.09.013

Nikolopoulou, K. (2020). Secondary education teachers' perceptions of mobile phone and tablet use in classrooms: benefits, constraints and concerns. Journal of Computers in Education, 10(2), 257-275. https://www.springerprofessional.de/en/secondary-education-teachersperceptions-of-mobile-phone-and-tab/17665804

Organización de las Naciones Unidas. (2014). Derechos de la infancia en la era digital. Desafíos: Boletín de la infancia y adolescencia sobre el avance de los objetivos de desarrollo del milenio. Derechos de la infancia en la era digital. http://repositorio.cepal.org/handle/11362/37139

Organización de las Naciones Unidas para la Educación, la Ciencia y la Cultura [UNESCO]. (2017). Informe de los Objetivos de Desarrollo Sostenible 2017. https://doi.org/10.18356/70388b69-es 
Pramuda, A., Mundilarto, Kuswanto, H. \& Hadiati, S. (2019). Effect of realtime physics organizer based smartphone and indigenous technology to students' scientific literacy viewed from gender differences. International Journal of Instruction, 12(3), 253-270. https://doi. org/10.29333/iji.2019.12316a

Román, C. A. (2017). El uso del celular y su influencia en las actividades académicas y familiares de los estudiantes de primer año de bachillerato de la Unidad Educativa Sagrados Corazones de Rumipamba de la ciudad de Quito. (Tesis de maestría inédita). Universidad Andina Simón Bolívar, Ecuador. https://core.ac.uk/download/pdf/160741743.pdf

Sirakaya, M. \& Kiliç Çakmak, E. (2017). Investigating Student Attitudes towards Augmented Reality. Malaysia Online Journal of Educational Techology, 6(1), 30-44. https://files.eric.ed.gov/fulltext/EJ1165447.pdf

Soriano, A. G. (2018). Dispositivos Móviles. Revista.seguridad.unam.mx, 7. https://revista.seguridad.unam.mx/numero-07/dispositivos-moviles

Stake, R. E. (2010). Qualitative Research: Studying How Things Work. New York, NY: Guilford Press.

Urrútia, G. \& Bonfill, X. (2010). PRISMA declaration: A proposal to improve the publication of systematic reviews and meta-analyses. Medicina Clinica, 135(11), 507-511. https://doi.org/10.1016/j.medcli.2010.01.015

Vázquez Calvo, B. \& Cassany, D. (2016). Language learning actions in two 1x1 secondary schools in Catalonia: the case of online language resources. New perspectives on teaching and working with languages in the digital era, 73-82. https://doi.org/10.14705/rpnet.2016.tislid2014.423

Villani, D., Morganti, L., Carissoli, C., Gatti, E., Bonanomi, A., Cacciamani, S., \& Riva, G. (2018). Students' acceptance of tablet PCs in Italian high schools: Profiles and differences. British Journal of Educational Technology, 49(3), 533-544. https://doi.org/10.1111/bjet.12591 
Xie, H., Peng, J., Qin, M., Huang, X., Tian, F. y Zhou, Z. (2018). Can touchscreen devices be used to facilitate young children's learning? A meta-analysis of touchscreen learning effect. Frontiers in Psychology, 9, 1-15. https:// doi.org/10.3389/fpsyg.2018.02580 